\title{
Enunciación
}

http://revistas.udistrital.edu.co/ojs/index.php/enunc

DOI: http://dx.doi.org/10.14483/udistrital.jour.enunc.2016.2.a08

\section{La comunicación científica oral de los estudiantes de Medicina Veterinaria y Zootecnia de la Universidad Central "Marta Abreu" de Las Villas}

\section{The oral scientific communication students of Veterinary Medicine of the Central University "Marta Abreu" of Las Villas}

\author{
Darlén Méndez Lloret ${ }^{1}$, Dalila Rodríguez González², Silvia Alonso Paz³, \\ María del Carmen Navarrete Reyes ${ }^{4}$
}

Para citar este artículo: Méndez, D.; Rodríguez, D.; Alonso, S. y Navarrete M. (2016). La comunicación científica oral de los estudiantes de Medicina Veterinaria y Zootecnia de la Universidad Central "Marta Abreu" de Las Villas. Enunciación, 21(2), $288-295$.

Recibido: 28-abril-2016 / Aprobado: 7-septiembre-2016

\section{Resumen}

El desarrollo de la comunicación científica es un elemento básico para todos los estudiantes universitarios en la actualidad, pues la dinámica de la sociedad requiere comunicar con coherencia y cohesión los resultados de la investigación. El objetivo del artículo es socializar la experiencia de cómo potenciar la comunicación científica oral en los estudiantes de Medicina Veterinaria y Zootecnia de la Universidad Central "Marta Abreu" de Las Villas. Para el desarrollo del trabajo se utilizaron métodos del nivel empírico como la observación y la revisión bibliográfica. Se realizó una prueba pedagógica de entrada a los estudiantes de primer y cuarto años que cursaban la carrera de Medicina Veterinaria y Zootecnia en dicha universidad, en el curso académico 2013-2014; esta evidenció carencias en la comunicación científica oral de los estudiantes. Para erradicar los problemas detectados se elaboraron e implementaron acciones didácticas que perfeccionaran este tipo de comunicación en los estudiantes muestreados. Actualmente los estudiantes valoran de manera positiva la importancia social y cultural que tiene en la actividad científico-investigativa la comunicación científica oral.

Palabras clave: comunicación, estudiante universitario, medicina veterinaria.

\begin{abstract}
Actually, the development of the scientific communication is a main element for all university students, the dynamics of the company needs to communicate the research findings with coherence and cohesion. The aim of this article is to socialize the experience of how promoting the oral scientific communication in the faculty of Veterinary Medicine of the Central University "Marta Abreu" of Las Villas. In the past, the observation and bibliographical review were used as empirical methods. One experiment
\end{abstract}

Universidad Central "Marta Abreu" de Las Villas. Correo electrónico: darlenm@uclv.edu.cu

2 Universidad Central "Marta Abreu" de Las Villas. Correo electrónico: dalila@uclv.edu.cu

3 Universidad Central "Marta Abreu" de Las Villas. Correo electrónico: silviaa@uclv.edu.cu

4 Universidad Central "Marta Abreu" de Las Villas. Correo electrónico: mcnavarr@uclv.edu.cu 
was done with the first and four year students who belong to this faculty from this university, as a result the students lacks oral scientific communication. To mitigate the detected issues were made and implemented by didactic actions that were improved this kind of communication

\section{INTRODUCCIÓN}

En la Universidad Central "Marta Abreu" de Las Villas se ha implementado un grupo de acciones para la formación en comunicación científica de los universitarios, entre las más importantes se encuentran: la elaboración de un programa para el desarrollo de la comunicación científica escrita en profesionales de Agronomía; la realización de una tesis doctoral sobre la articulación de la enseñanza del idioma ruso con la física, la química y la matemática en el plano lexical; la realización de un trabajo investigativo sobre las marcas de conexión discursiva en los textos científicos, de forma conjunta con la Universidad Nacional de Catamarca (Argentina); la elaboración de indicadores para evaluar la producción científica escrita en la enseñanza posgraduada; la impartición de la asignatura "Taller de Comunicación Científica" en varias carreras; la investigación sobre juego de roles profesionales para desarrollar el discurso oral científico y la generalización del proyecto institucional: "Estrategia didáctica para potenciar en los profesionales e investigadores habilidades comunicativas para la comunicación científica".

Existen referencias internacionales como Sánchez (1984); Molestina (1987,1988); Cassany, Luna y Sanz (1993); Van Dijk (1997); Gutiérrez (1998); Rizo (2006); entre otros; además autores nacionales como Mesa (2003), Roméu (2005, 2011); Navarrete, Morales y Rodríguez (2010); Méndez (2014), y Rodríguez (2014), cuyos resultados de investigación ofrecen importantes aportes metodológicos sobre la comunicación, la comunicación oral y la comunicación científica. in the sample students. Nowadays, the students have a good perception of the social and cultural importance that scientist performs in the oral scientific communication.

Keywords: communication, university students, veterinary medicine.

Los aportes de estos autores y las acciones antes mencionadas han contribuido positivamente desde la teoría y la práctica al desarrollo de las habilidades comunicativas para la comunicación científica en los universitarios; no obstante, los estudiantes universitarios aún tienen carencias en cuanto a la comunicación científica oral, una de las causas es la desatención a la temática, por lo cual se debe reconocer que se ha trabajado más la escritura científica.

En el mundo se le ha concedido prioridad al tratamiento de la comunicación científica fundamentalmente en los sistemas de enseñanza, pero en muchas ocasiones no ha surtido el efecto que se quiere. Desde los primeros niveles o grados de la enseñanza primaria y hasta la universitaria, los estudiantes necesitan desarrollar el habla, la escritura, la lectura, la audición y la interacción, como habilidades básicas del proceso comunicativo, así lograrían ser profesionales competentes en su lengua materna. Es importante que el estudiante utilice correctamente el lenguaje científico porque la producción de textos en el área de la ciencia requiere de procesos indispensables para el desarrollo de la comunidad científica y de la sociedad.

La comunicación científica es el proceso de transmisión y difusión del conocimiento, la forma a través de la que se incorporan al saber humano los resultados derivados de la actividad investigativa. Ello es trascendente por la falsación a la que obligatoriamente debe someterse todo nuevo conocimiento y por la naturaleza reproductible que tiene la ciencia. Lo primero tiene que ver con la ineludible licitación que debe tener el conocimiento generado por un investigador ante el gremio científico; lo segundo con la idea de que se genera ciencia a partir de ciencia (VillabeIla, 2012, p. 111). 
No se debe descuidar por parte de profesores y estudiantes universitarios el trabajo con la comunicación oral, que tan lacerada está en los tiempos actuales. Esta forma de comunicación es de gran importancia cuando se transmite información reciente que no se ha procesado o cuando los resultados científicos son novedosos y aún no se han escrito. El conocimiento científico se comunica de forma escrita y oral. La primera se realiza a través de artículos, ensayos, informes, etc., los que por su naturaleza son más perdurables. La segunda se desarrolla mediante conferencias, disertaciones, ponencias, pósteres, etc., ante un auditorio delimitado, por lo que su trascendencia es más efímera.

La comunicación científica es el proceso de interacción que se produce entre los individuos que conforman una comunidad científica, y que al estar dirigida a la producción y distribución de los conocimientos científicos debe compartir las características de la ciencia: objetividad, rigurosidad y sistematicidad, las que, mediante el lenguaje científico se plasman en el texto científico (Rodríguez, 2014, p. 15).

La comunicación oral en el contexto científico se trasmite por el canal de la oralidad y los elementos que lo conforman son: el canal auditivo, el contexto extralingüístico, la interacción, los códigos no verbales y los signos lingüísticos de manera sucesiva e inmediata.

La comunicación científica informal u oral es aquella que se realiza de manera verbal ante un auditorio al cual se exponen los resultados parciales o finales de una investigación, o se razona sobre un tema científico. Es una forma de transmitir la ciencia más efímera que la escrita por la finitud de la concurrencia y la fugacidad de sus efectos (Villabella, 2012, p. 146).

Los estudiantes de la carrera de Medicina Veterinaria y Zootecnia, al igual que el resto de los estudiantes universitarios, exponen sus resultados científicos mediante la ponencia como género más importante y más utilizado de la comunicación científica oral. Estos resultados no siempre son comunicados de manera coherente debido a las insuficiencias que poseen en este tipo de comunicación. En este reporte de caso los estudiantes universitarios muestreados elaboraron ponencias como género fundamental de la comunicación científica oral.

Todo médico veterinario presenta un diagnóstico de caso, explica la enfermedad que ataca al animal que esté analizando y ofrece el tratamiento correspondiente. Estas actividades son expuestas por ellos mediante trabajos de curso, trabajos de diploma, ponencias en eventos científicos estudiantiles; a través de ellas se deben comunicar los resultados científicos de manera lógica, fluida y exacta.

Por ello las autoras se acogen a la definición de que "la comunicación científica oral esla forma de la comunicación científica que emplean los miembros de una comunidad científica para socializar oralmente de manera exacta y fluida los resultados obtenidos en el proceso de investigación" (Méndez, 2014, p. 15).

Derivado de lo planteado en este artículo,se presenta el siguiente problema: ¿Cómo desarrollar la comunicación científica oral de los estudiantes de Medicina Veterinaria y Zootecnia de la Universidad Central "Marta Abreu" de Las Villas? Para resolver dicho interrogante se expresa el siguiente objetivo: elaborar acciones didácticas para la potenciación de la comunicación científica oral de los estudiantes de Medicina Veterinaria y Zootecnia de dicha institución.

\section{MATERIALES Y MÉTODOS}

En el curso académico 2013-2014 a los estudiantes de primer y cuarto años de la carrera Medicina Veterinaria y Zootecnia se les aplicó una prueba pedagógica de entrada con la finalidad de constatar las fortalezas y debilidades que tenían en cuanto a su lengua materna. Los resultados evidenciaron que los estudiantes presentaban 
insuficiencias en cuanto a la comunicación científica oral, particularmente en la ponencia como género discursivo.

Después de analizar estos resultados se llegó a la conclusión de que los estudiantes necesitan indicadores para la evaluación de su comunicación científica oral. Una vía para el logro de la apropiación de estos conocimientos es mediante la asignatura de "Lengua Materna".

Se adelantaron acciones didácticas a partir de las cuales se utilizaron métodos del nivel empírico como la observación y la revisión bibliográfica. Se utilizó la metodología cualitativa y se puso en práctica el diario de campo. La muestra escogida fue de 100 estudiantes de primer y cuarto años de la carrera de Medicina Veterinaria y Zootecnia de la Facultad de Ciencias Agropecuarias, lo que representó el $83,3 \%$ del total de estudiantes de esos años. Se trabajó con una muestra intencional. Se observaron 3 actividades científicas donde participaron los estudiantes a lo largo del segundo semestre y se grabaron las exposiciones de los mismos.

La elaboración de indicadores para la evaluación de la comunicación científica oral de los estudiantes de Medicina Veterinaria y Zootecnia fue una de las herramientas didácticas que se desarrollaron. En el ámbito internacional no existe una definición oficial sobre el término indicador. Sí hay diversos conceptos y definiciones con respecto al tema.

Según Beltrán (s.f., p. 1) un indicador es "la relación entre las variables cuantitativas o cualitativas, que permite observar la situación y las tendencias de cambio generadas en el objeto o fenómeno observado, respecto de objetivos y metas previstas e influencias esperadas".

Las autoras se acogen a este criterio porque el estudiante de Medicina Veterinaria y Zootecnia debe estar en la capacidad de transformar su comunicación científica oral, debe comparar su situación actual con los resultados que desea alcanzar y debe discernir entre el retroceso y el avance con respecto al tema.
A continuación se enuncian los indicadores elaborados para la evaluación de la comunicación científica oral. Estos se relacionan con las operaciones cognitivas del proceso de oralidad: planificación, producción y corrección.

\section{Indicadores (etapa de planificación)}

- Estado de la presentación.

- Medio audiovisual.

- Estructura de la presentación.

- Plan de la presentación.

\section{Indicadores (etapa de producción)}

- Lenguaje verbal.

- Lenguaje no verbal.

- Terminología científico-técnica.

- Vocabulario.

- Imagen auditiva.

- Idioma.

- Morfosintaxis.

- Conducción y/o interacción.

- Coherencia y cohesión de las ideas.

- Comprensión por parte del auditorio.

- Resumen y repetición de ideas importantes.

- Edición.

\section{Indicadores (etapa de corrección)}

- Autocorrección y/o corrección de la coherencia y cohesión de las ideas.

- Generación de nuevas ideas.

- Eliminación o agregación de ideas.

- Comparación de las nuevas ideas con las anteriores.

- Reformulación de ideas.

- Constatación del registro adecuado.

- Autocorrección y/o corrección de la terminología científica adecuada.

- Cumplimiento del plan de la presentación.

- Cumplimiento de la estructura de la presentación. 


\section{RESULTADOS Y DISCUSIÓN}

La profesora de la asignatura "Lengua Materna" participó en tres actividades científicas en las cuales los estudiantes expusieron los resultados de investigaciones que realizaban, luego de observar estas actividades, teniendo en cuenta las etapas y los indicadores elaborados, se muestran las siguientes regularidades:

En la primera actividad científica la etapa de planificación se vio afectada porque la mayoría de los estudiantes carecían de un buen estado de la presentación, no tenían definido el medio audiovisual que utilizarían, la estructura de la presentación estaba incompleta, al igual que el plan de la presentación. En cuanto a la etapa de producción, la mayoría de los estudiantes no le dio el uso adecuado al lenguaje verbal y al lenguaje no verbal; la terminología científico-técnica y el vocabulario no fueron correctos; la imagen auditiva no fue utilizada adecuadamente así como el idioma y la morfosintaxis; la conducción o interacción, la coherencia y cohesión de las ideas, el resumen y repetición de ideas importantes no fueron efectivos por parte de la mayoría de los estudiantes, y por ende la comprensión por parte del auditorio y la edición no fueron las mejores. En la etapa de corrección estos estudiantes no lograron: la autocorrección o corrección de la coherencia y cohesión de las ideas, la generación de nuevas ideas, la eliminación o agregación de ideas, la comparación de las nuevas ideas con las anteriores, la reformulación de ideas, la constatación del registro adecuado, la autocorrección o corrección de la terminología científica adecuada; el cumplimiento del plan de la presentación y el de su estructura. En resumen, la evaluación dada al $90 \%$ de los estudiantes fue de mal; el $10 \%$ de los estudiantes fue evaluado de regular.

Se comenzó a estudiar por parte de los estudiantes y a explicar por parte de la profesora los indicadores con el fin de lograr el perfeccionamiento en la comunicación científica oral, los estudiantes comenzaron a apropiarse del contenido y a aplicarlo con vistas a realizar exposiciones científicas coherentes, fluidas, exactas y cohesionadas, mediante la ponencia como género discursivo más importante.

En la segunda actividad científica se demostró el progreso de los estudiantes en comparación con la primera actividad, pues los resultados mejoraron considerablemente. Durante las clases de "Lengua Materna" se efectuaron talleres para potenciar espacios de debate en los cuales los estudiantes comprendieron mejor los ejemplos que se seleccionaron de la práctica, estos enriquecieron la experiencia vivida.

En la etapa de planificación, los indicadores más afectados fueron el medio audiovisual y la estructura de la presentación. En la etapa de producción los más perjudicados fueron la terminología científico-técnica y el vocabulario, la morfosintaxis, la conducción o interacción, la coherencia y cohesión de las ideas, la comprensión por parte del auditorio. En la etapa de corrección los estudiantes no lograron la autocorrección o corrección de la coherencia y cohesión de las ideas, la constatación del registro adecuado, la autocorrección o corrección de la terminología científica adecuada y el cumplimiento de la estructura de la presentación. En resumen, la evaluación dada al $80 \%$ de los estudiantes fue de regular; el $20 \%$ de los estudiantes fue bien evaluado.

En la tercera actividad científica, el indicador más afectado de la etapa de planificación fue el medio audiovisual. En la producción, los indicadores más bajos fueron la terminología científico-técnica y el vocabulario. En la etapa de corrección, los indicadores más afectados fueron la autocorrección o corrección de la coherencia y cohesión de las ideas, y la autocorrección o corrección de la terminología científica. En resumen, la evaluación dada al 90 \% de los estudiantes fue buena, y solo el $10 \%$ obtuvo evaluación regular. Ninguno recibió una mala evaluación. 
La aplicación web para potenciar este tipo de comunicación fue otra acción didáctica adelantada. Su objetivo estuvo enfocado en el desarrollo de la producción de textos científicos orales con calidad mediante la ponencia. Para el diseño e implementación de esta aplicación se seleccionaron como lenguajes de programación el JavaScript y el CSS, como servidor Web el Apache y como sistema gestor de bases de datos el MySQL. Se utilizó el CMS Joomla como ambiente de desarrollo y se incluyeron módulos para soportar las funcionalidades como: carga y descarga de archivos y ejercicios para desarrollar la habilidad de expresión oral. La aplicación web estuvo al alcance de los estudiantes de Medicina Veterinaria y Zootecnia, en la intranet de la Universidad Central "Marta Abreu", de Las
Villas. La aplicación web fue utilizada por todos los estudiantes muestreados, y tuvo gran aceptación. En la figura 1 se muestra la interfaz de la aplicación web elaborada.

\section{CONCLUSIONES}

Se llevaron a cabo acciones didácticas para la potenciación de la comunicación científica oral de los estudiantes universitarios; ellos deben continuar trabajando con los indicadores más afectados: el medio audiovisual, la terminología científico-técnica, el vocabulario, la autocorrección o corrección de la coherencia y cohesión de las ideas, y la autocorrección o corrección de la terminología científica.

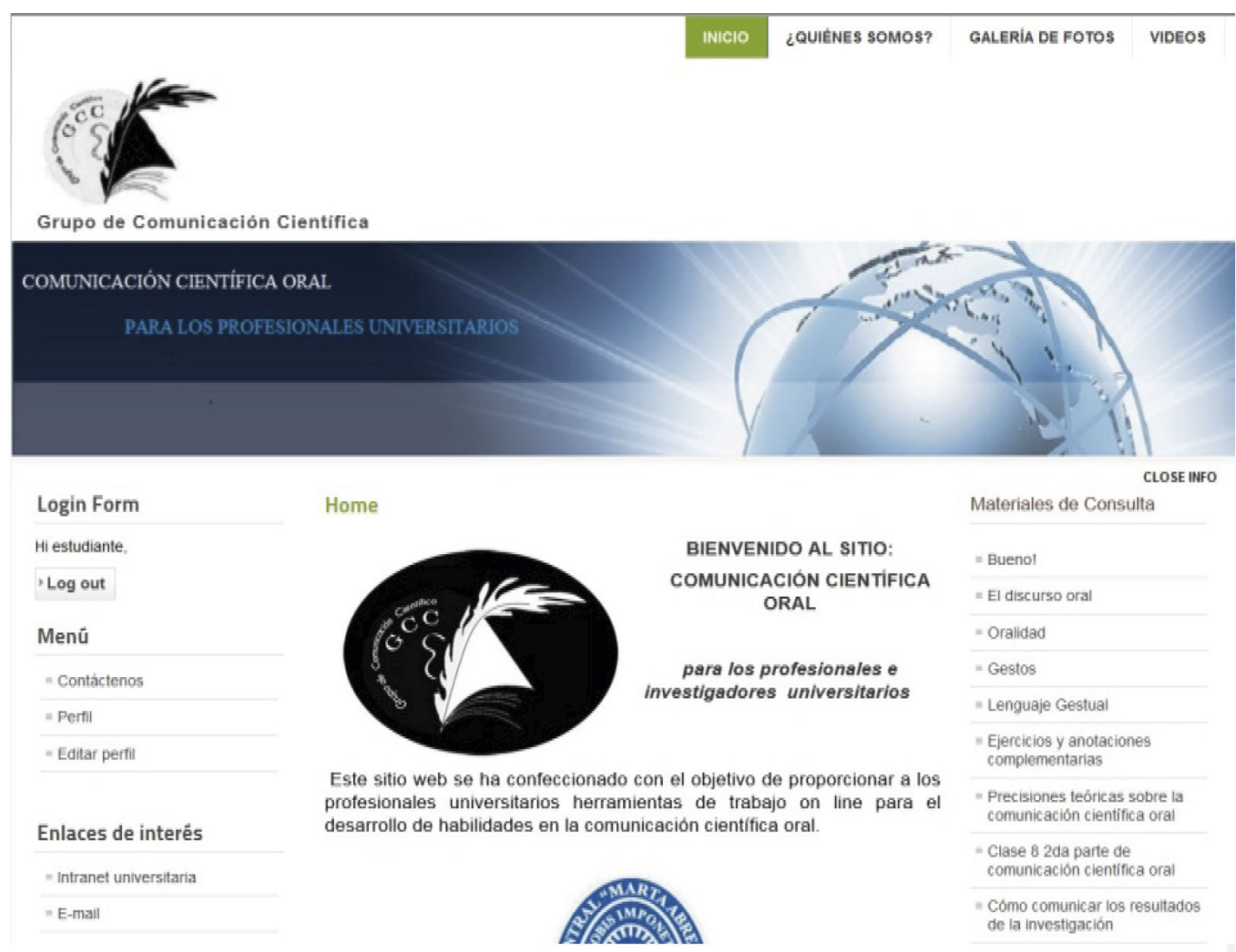

Figura 1. Aplicación web de comunicación científica oral

Fuente: Informe en opción al Premio CITMA Nacional del proyecto Estrategia linguodidáctica para potenciar en los profesionales la comunicación científica (2014). 
La implementación de acciones didácticas permitió el progreso de este tipo de comunicación tan vulnerada en la actualidad; de esta manera, todo estudiante puede utilizarlas para potenciar habilidades en la comunicación científica oral mediante la ponencia. Esto le permite conocer su estado actual y cómo llegar a lo deseado.

El colectivo pedagógico de los dos años muestreados trasmitió juicios a favor del desempeño de las acciones didácticas adelantadas, porque constituyen aportes enriquecedores al modelo del profesional de la carrera.

\section{RECONOCIMIENTOS}

Resultado del proyecto institucional "Formación en comunicación científica de los universitarios", perteneciente a la Universidad Central "Marta Abreu", de Las Villas.

Resultado parcial de la tesis doctoral "Estrategia didáctica para desarrollar la comunicación científica oral de los estudiantes de Medicina Veterinaria y Zootecnia de la Universidad Central "Marta Abreu" de Las Villas".

\section{REFERENCIAS BIBLIOGRÁFICAS}

Beltrán, J. (s.f.). Indicadores de gestión. Recuperado de http://www.iue.edu.co/documents/emp/comoGerenciar.pdf.

Campos, R. (2000). La comunicación científica: ¿Arte o ciencia? Granada: Art, Pharmeceutica.

Cassany, D.; Luna, M. y Sanz, G. (1993). Enseñar lengua. Barcelona: Graó.

Gutiérrez, B. (1998). La ciencia empieza en la palabra. Análisis e historia del lenguaje científico. Barcelona: Editorial Península.

Hernández, R.; Fernández, C. y Baptista, P. (1996). Metodología de la investigación. Distrito Federal: McGraw-Hill/Interamericana Editores, S.A. de CV.

Horacio, M. (2006). Dimensión ética de la comunicación científica y tecnológica. Ciudad de Buenos Aires: Instituto Nacional de Tecnología Agropecuaria.
Méndez, D. (2014). Informe en opción al Premio CITMA Nacional del proyecto Estrategia linguodidáctica para potenciar en los profesionales la comunicación científica. Santa Clara: Editorial Feijóo.

Mesa, M. (2003). Propuestas de programas de comunicación científica escrita para profesores de Agronomía. Tesis en opción al título de Máster en Educación. Santa Clara, VC, Cuba: Universidad Central "Marta Abreu" de Las Villas.

Molestina, C. et al. (1987). Fundamentos de comunicación científica y redacción técnica. Montevideo: Programa Cooperativo de Investigación Agrícola del Cono Sur. IICA-CIDIA.

Molestina, C. (1988). El método científico y el proceso de la comunicación. En: Fundamentos de la comunicación científica y redacción técnica. San José: Instituto Interamericano de Cooperación para la Agricultura. Recuperado de: http://repiica.iica.int/ docs/B2298e/B2298e.pdf.

Navarrete, M.; Morales, J. y Rodríguez, D. (2010). La evaluación de la comunicación científica escrita desde una perspectiva didáctica en la enseñanza posgraduada. Enunciación, 15, 95-104.

Rizo, M. (2006). Interacción y comunicación. Exploración teórica-conceptual del concepto de interacción. Recuperado de: www.monografias.com

Rodríguez, D. (2014). Informe en opción al Premio CITMA Nacional del proyecto Estrategia linguodidáctica para potenciar en los profesionales la comunicación científica. Santa Clara: Editorial Feijóo.

Roméu, A. (2005). El procesamiento de la información científico técnica y la producción de textos científicos como problema interdisciplinario. Ciudad de La Habana: IPLAC. Recuperado de http://biblioteca.uniss.edu.cu/sites/default/files/CD/pedagogia/ cursos/pdfp2005/Curso65.pdf

Roméu, A. (2011) La redacción de textos científicos. Revista Referencia Pedagógica. Ciudad de La Habana: Editorial CUJAE. Recuperado de: http://cujae.edu. cu/publicaciones/referencia/No4/seccion10.htm

Sánchez, R. (1984). La edición del texto oral. En: AISO. Actas I (pp. 69-77). Madrid: Centro Virtual Cervantes. Recuperado de: http://cvc.cervantes.es/literatura/aiso/pdf/01/aiso_1_007.pdf. 
Stuart, C. (1990). Técnicas básicas para hablar en público. Madrid: Ediciones Deusto.

Torrent, M. (2004). La oralidad ¿Se enseña a hablar y a escuchar en la escuela? Revista Intertexto, 3(5). Recuperado de: http://www.sagrado.edu.ar/def/ revista-intertexto.
Van Dijk, T. (1997). La ciencia del texto. Madrid: Editorial Paidós. Recuperado de http://www.discursos.org/oldbooks/Teun\%20A\%20van\%20Dijk\%20 -\%20La\%20Ciencia\%20del\%20Texto.pdf

Villabella, C. (2012). Investigación y comunicación científica en la ciencia jurídica. La Habana: Editorial Universitaria Félix Varela.

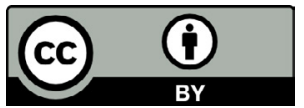

